\title{
The Time Dependent Amplitude Equation for the Swift-Hohenberg Problem
}

\author{
P. Collet ${ }^{1}$ and J.-P. Eckmann ${ }^{2}$ \\ ${ }^{1}$ Centre de Physique Théorique, Laboratoire UPR A14, CNRS, Ecole Polytechnique, \\ F-91128 Palaiseau, France \\ ${ }^{2}$ Département de Physique Théorique, Université de Genève, $\mathrm{CH}-1211$ Geneva 4, Switzerland
}

\section{Dedicated to Res Jost and Arthur Wightman}

\begin{abstract}
Precise estimates for the validity of the amplitude approximation for the Swift-Hohenberg equation are given, in a fully time dependent framework. It is shown that small solutions of order $\mathcal{O}(\varepsilon)$ which are modulated like stationary solutions have an evolution which is well described in the amplitude approximation for a time of order $\mathcal{O}\left(\varepsilon^{-2}\right)$. For the proofs, we use techniques for nonlinear semigroups and oscillatory integrals.
\end{abstract}

\section{Introduction}

In this paper, we study the relation between a multi-scale nonlinear problem and its associated amplitude equation in a fully time-dependent framework. In order to keep the exposition sufficiently simple, we state and prove our results in the framework of the Swift-Hohenberg equation

$$
\partial_{t} u(x, t)=\left(3 \varepsilon^{2}-\left(1+\partial_{x}^{2}\right)^{2}\right) u(x, t)-u^{3}(x, t) .
$$

Here, $u$ is a function $\mathbf{R} \times \mathbf{R}^{+} \rightarrow \mathbf{R}$. This equation has been studied in detail in [1], and we summarize those of the results which are relevant for the current study.

1. Equation (1.1) has stationary (i.e., time-independent) solutions, for small $\varepsilon$ which are of the form

$$
u(x, t) \approx 2 \varepsilon \cos (x) .
$$

2. Equation (1.1) has front solutions which are of the form

$$
u(x, t)=\varepsilon \sum_{n \in \mathbf{Z}} u_{n}\left(\varepsilon x-\varepsilon^{2} c t\right) e^{i n x},
$$

with the reality conditions

$$
u_{n}(y)=\bar{u}_{-n}(y)
$$


The solutions are front solutions because the amplitudes $u_{n}$ can be chosen to be zero for $y \rightarrow \infty$ and nonzero for $y \rightarrow-\infty$. In fact, the dominating terms in the sum above are the ones with $u_{ \pm 1}$, and $u_{ \pm 1}$ is of order $\mathcal{O}(1)$ while all other $u_{n}$ are at least of order $\mathcal{O}(\varepsilon)$.

3. A multiscale analysis shows that the amplitude $u_{1}$ satisfies approximately the amplitude equation

$$
\left(4 \partial_{x}^{2}+c \partial_{x}+3\right) u_{1}-3 u_{1}\left|u_{1}\right|^{2}=0 .
$$

The term with $c \partial_{x}$ is generated by considering the $u_{n}$ in a frame moving with speed $\varepsilon^{2} c$.

All the solutions above can be considered to be quasistationary, i.e., constant in some frame of reference, or, for the case of front solutions, having constant envelopes in some frame of reference. Therefore, they are amenable to a lowdimensional study, which has been detailed in [1] and in [2].

In the present paper, we study the more complicated question of "full" timedependence. That is, we do not restrict our attention to a submanifold of special solutions but instead ask for the time evolution of certain types of initial conditions. It is a general principle of physics that in situations of the type described above the "full" evolution is - for small $\varepsilon$ - still accurately described by the evolution of the amplitude through the amplitude equation (1.3), in which the $\varepsilon$-dependent terms have been dropped. Here, we describe in detail the nature of this approximation, and give bounds on its regime of validity. To make this comparison more precise, we rewrite the function $u(x, t)$ in the form

$$
u(x, t)=\varepsilon v\left(\varepsilon x, \varepsilon^{2} t\right) e^{i x}+\text { complex conjugate, }
$$

and we assume $v_{0}(x)=v(x, 0)$ is given. In terms of $v$, the evolution equation (1.1) reads

$$
\partial_{t} v=\left(4 \partial_{x}^{2}+3-4 i \varepsilon \partial_{x}^{3}-\varepsilon^{2} \partial_{x}^{4}\right) v-3 v|v|^{2}-e^{2 i x / \varepsilon} v^{3} .
$$

Let us call the corresponding time evolution $T_{t}$. We want to compare it with the time evolution $S_{t}$ for the amplitude equation

$$
\partial_{t} w=\left(4 \partial_{x}^{2}+3\right) w-3 w|w|^{2}
$$

and ask how the difference $T_{t} v_{0}-S_{t} v_{0}$ evolves in time.

Our result is the

Theorem 1.1. Consider any function $v_{0}$ such that $\partial_{x}^{k} v_{0}$ is in $L^{\infty}$ for $k=0, \ldots, 4$. There is a $T>0$, an $\varepsilon_{0}>0$ and $a C<\infty$ such that for all $t \in(0, T]$ and all $\varepsilon \in\left[0, \varepsilon_{0}\right]$ one has,

$$
\left\|T_{t} v_{0}-S_{t} v_{0}\right\|_{\infty} \leqq C \min \left(\varepsilon t^{1 / 2}, \varepsilon^{1 / 2} t^{3 / 4}\right) \text {. }
$$

Remark. The constant $C$ only depends on $\left\|\partial_{x}^{k} v_{0}\right\|$, for $k=0, \ldots, 4$. The solution itself can be shown to exist already when one only assumes $v_{0}$ in $L^{\infty}$. However, the bounds will then show slight divergences near $t=0$, because of the time which is needed until the evolution has smoothed the initial data.

It is instructive to translate Theorem 1.1 back to the original equation (1.1), and the original time scale. Denote by $\widehat{T}_{t}$ the time evolution defined by (1.1), and let $v_{0} \in L^{\infty}$ be given. Define

$$
u_{0}(x)=\left(\mathscr{P} v_{0}\right)(x) \equiv \varepsilon\left(v_{0}(\varepsilon x) e^{i x}+\bar{v}_{0}(\varepsilon x) e^{-i x}\right) .
$$


With this definition, one has the relation

$$
\hat{T}_{t} \mathscr{P}=\mathscr{P} T_{t \varepsilon^{2}} .
$$

Thus we find,

Corollary 1.2. Consider any function $v_{0}$ such that $\partial_{x}^{k} v_{0}$ in $L^{\infty}$ for $k=0, \ldots, 4$. There is $a T>0$ and an $\varepsilon_{0}>0$ such that for all $\varepsilon \in\left[0, \varepsilon_{0}\right]$ and all $t \in\left(0, T / \varepsilon^{2}\right]$ one has,

$$
\left|\widehat{T}_{t} u_{0}(x)-\varepsilon e^{i x}\left(S_{t \varepsilon^{2}} v_{0}\right)(\varepsilon x)-\varepsilon e^{-i x}\left(S_{t \varepsilon^{2}} v_{0}\right)^{-}(\varepsilon x)\right| \leqq \varepsilon^{3} C \min \left(t^{1 / 2}, t^{3 / 4}\right) .
$$

Note that if $\|v\|_{\infty}=\mathcal{O}(1)$, then $\|\mathscr{P} v\|_{\infty}=\mathcal{O}(\varepsilon)$, so that Corollary 1.2 gives interesting bounds for solutions of size $\varepsilon$. This is the size of stationary solution of (1.1), cf. (1.2).

The difficulty in the proof of Theorem 1.1 is related to the fact that two seemingly contradictory arguments are used to eliminate the correction terms: For the differential operators we shall use a study of the semigroup they generate while for the high frequency part $e^{2 i x / \varepsilon}$, we use WKB-like methods, which need smoothness. This smoothness will only appear after a short time and a careful study of local singularities is necessary. This is responsible for the bound (1.6), while the reader might have expected $\mathcal{O}(\varepsilon t)$ instead.

The transformation $\mathscr{P}$ can be viewed as a zero order approximation to the problem of finding a normal form for the Swift-Hohenberg equation. The general problem can be formulated as follows: We are looking for a transformation $\mathscr{P}_{\varepsilon}$ which satisfies approximately

$$
D \mathscr{P}_{\varepsilon} \cdot Y=X \mathscr{P}_{\varepsilon}
$$

where

$$
\begin{gathered}
X(u)(x, t)=\left(3 \varepsilon^{2}-\left(1+\partial_{x}^{2}\right)^{2}\right) u(x, t)-u^{3}(x, t) \\
Y(v)(x, t)=\left(4 \partial_{x}^{2}+3\right) v(x, t)-3 v|v|^{2}(x, t)
\end{gathered}
$$

In this paper, we find the lowest order approximation, $\mathscr{P}$, to $\mathscr{P}_{\varepsilon}$ which is given by (1.7).

\section{A General Bound on Semigroups}

The main purpose of this paper is a comparison of semigroups. We want to show that they differ little, and we now explain the main techniques with which these differences are estimated. Since some of the perturbations have high frequency components as $\varepsilon \rightarrow 0$, we need to prove sufficient differentiability at every stage of the estimates.

The basic technique to do these estimates will always be the same, but the details will vary sufficiently at every stage of the proof to make a separate treatment necessary. To guide the reader, we therefore state and prove a "generic" statement which is a typical example of our technique. It is a straightforward generalization of the classical methods, cf. e.g., Lang [3], to the case of "semiflows" with unbounded generator. 
Our general setting takes place in a Banach space $\mathscr{B}$, and we assume that we are given a "free," possibly time-dependent, evolution operator $S_{t, t_{0}}$ which satisfies $S_{t, t}=1, S_{t, t^{\prime}} S_{t^{\prime}, t^{\prime \prime}}=S_{t, t^{\prime \prime}}$, for $t \geqq t^{\prime} \geqq t^{\prime \prime}$, and for which the derivative below exists:

$$
\partial_{t} S_{t, t_{0}}=L_{t} S_{t, t_{0}} \text {. }
$$

We want to solve an "interacting" problem

$$
\partial_{t} v_{t}=L_{t} v_{t}+F_{t}+G_{t} v_{t}+\mathcal{N}_{t}\left(v_{t}\right) .
$$

Here, we look for the unknown function $t \mapsto v_{t}$ as a map from $\mathbf{R}^{+}$to $\mathscr{B}$. The intuitive interpretation of the various terms in (2.1) is as follows: $L_{t}$ is the (timedependent) generator of the free evolution; $F_{t}$ is an inhomogeneity; $G_{t}$ is a linear operator (or antilinear or a sum of these); $\mathscr{N}_{t}$ is a nonlinearity.

The formal solution (2.1) with initial data $v_{0}$ is given by

$$
v_{t}=S_{t, 0} v_{0}+\int_{0}^{t} d \tau S_{t, \tau}\left(F_{\tau}+G_{\tau} v_{\tau}+\mathscr{N}_{\tau}\left(v_{\tau}\right)\right)
$$

Typical assumptions on the various terms in (2.1) look as follows. Denote by $\|\cdot\|$ the norm in $\mathscr{B}$. Then

$$
\begin{aligned}
& A_{S}:\left\|S_{t, \tau} v\right\| \leqq s(t, \tau)\|v\|, \\
& A_{F}:\left\|F_{\tau}\right\| \leqq f(\tau), \\
& A_{G}:\left\|G_{\tau} v\right\| \leqq g(\tau)\|v\|, \\
& A_{N}:\left\|\mathcal{N}_{\tau}(v)\right\| \leqq n(\tau)\|v\| \cdot h(\|v\|), \text { where } h(\varrho) \leqq \min \left(1, \varrho^{v}\right) \text { for some } v>0 .
\end{aligned}
$$

We shall also need some sort of control on the functional derivative of the nonlinearity $\mathscr{N}$ (the other terms have obvious functional derivatives). $A_{N}^{\prime}:\left\|\mathscr{N}_{\tau}(v)-\mathscr{N}_{\tau}\left(v^{\prime}\right)\right\| \leqq m(\tau)\left\|v-v^{\prime}\right\| \cdot k\left(\|v\|+\left\|v^{\prime}\right\|\right)$, where $k(\varrho) \leqq \min \left(1,(\varrho / 2)^{v^{\prime}}\right)$, for some $v^{\prime} \geqq 0$.

We shall now study Eq. (2.2) by considering a space $\mathbf{B}$ of functions $\mathbf{R}^{+} \rightarrow \mathbf{B}$. More precisely, given some "final time" $T>0$, and a positive weight function $b:(0, T] \rightarrow \mathbf{R}^{+}$we define

$$
\mathbf{B}=\left\{\left\{v_{\tau}\right\}_{\tau \geqq 0} \mid \sup _{T \geqq \tau>0} b(\tau)\left\|v_{\tau}\right\|<\infty\right\}
$$

and we equip $\mathbf{B}$ with the norm

$$
\|v\|\left\|=\sup _{T \geqq \tau>0} b(\tau)\right\| v_{\tau} \| .
$$

Remark. In applications of this general scheme, the norm $\|\cdot\|$ could contain spatial derivatives of $v$ and the weight $b$ could be chosen with a (mild) singularity at zero to take into account the time scale on which solutions get smooth.

In order to study Eq. (2.2) we consider a map $\mathscr{M}: \mathbf{B} \rightarrow \mathbf{B}$, defined by

$$
(\mathscr{M} v)_{t}=S_{t, 0} v_{0}+\int_{0}^{t} d \tau S_{t, \tau}\left(F_{\tau}+G_{\tau} v_{\tau}+\mathscr{N}_{\tau}\left(v_{\tau}\right)\right) .
$$


If $\mathscr{M} v=v$, then $v_{\tau}$ is a solution of (2.1) with initial data $v_{0}$. It is useful to consider in $\mathbf{B}$ the affine subspace $\mathbf{B}_{w}$, with $w \in \mathscr{B}$, defined by

$$
\mathbf{B}_{w}=\left\{v \in \mathbf{B} \mid v_{0}=w\right\} .
$$

Clearly, $\mathbf{B}_{w}$ is the set of functions with identical initial data $v_{0}=w$. To show that $\mathscr{M} v=v$ has a solution in $\mathbf{B}_{w}$, we show that $\mathscr{M}$ is a contraction of a ball in $\mathbf{B}$. Let $\mathbf{B}^{A}=\{v \in \mathbf{B},\|v\| \|<A$, and assume that $A$ is so small that $A / b(\tau)<1$, for $\tau \in(0, T]$. This condition will make our estimates on $\mathscr{M}$ simpler, but is otherwise inessential. In this case, we always find $h(\|v\|) \leqq\|v\|^{v}$ for $v \in \mathbf{B}^{A}$. The other case is then handled analogously. By our assumptions we get

$$
\begin{aligned}
b(t)\left\|(\mathscr{M} v)_{t}\right\| \leqq & b(t) s(t, 0)\left\|v_{0}\right\| \\
& +\int_{0}^{t} d \tau b(t) s(t, \tau)\left(f(\tau)+g(\tau) A / b(\tau)+n(\tau)(A / b(\tau))^{1+\nu}\right) .
\end{aligned}
$$

In order to make the estimates more transparent, we define the constants

$$
\begin{aligned}
& S=\sup _{T \geqq t>0} s(t, 0) b(t), \\
& F=\sup _{T \geqq t>0} \int_{0}^{t} d \tau b(t) s(t, \tau) f(\tau), \\
& G=\sup _{T \geqq t>0} \int_{0}^{t} d \tau b(t) s(t, \tau) g(\tau) / b(\tau), \\
& N=\sup _{T \geqq t>0} \int_{0}^{t} d \tau b(t) s(t, \tau) n(\tau) / b(\tau)^{1+v} .
\end{aligned}
$$

With these notations, a sufficient condition for $b(t)\left\|(\mathscr{M} v)_{t}\right\| \leqq A$ to hold is

$$
S\left\|v_{0}\right\|+F+G A+N A^{1+v} \leqq A .
$$

This is possible whenever

$$
G<1,
$$

$$
\left(S\left\|v_{0}\right\|+F\right) N^{1 / v} \leqq(1-G)^{(1+v) / v}\left(\frac{1}{(1+v)^{1 / v}}-\frac{1}{(1+v)^{(1+v) / v}}\right) .
$$

The condition (2.5) says that the linear part of the perturbation is small and the condition (2.6) can be satisfied for small initial data and small inhomogeneities.

Proof of Sufficiency of (2.6). Define $x=A N^{1 / v}$. Then (2.4) says

$$
\left(S\left\|v_{0}\right\|+F\right) N^{1 / v}+x^{1+v} \leqq(1-G) x .
$$

The difference of the two sides of (2.7) attains a minimum as a function of $x$ when the derivatives coincide, i.e., when

$$
(1+v) x^{v}=(1-G), \text { i.e., } \quad x=((1-G) /(1+v))^{1 / v} .
$$

The difference is then seen to be non-negative if (2.6) holds.

Thus: if (2.5) and (2.6) hold, then $\mathscr{M}$ maps a ball of radius $A$ in $\mathbf{B}$ into itself. We now work out a condition which insures that $\mathscr{M}$ is a contraction on $\mathbf{B}_{w}$, for 
sufficiently small $\|w\|$. By definition,

$$
(\mathscr{M} v)_{t}-\left(\mathscr{M} v^{\prime}\right)_{t}=\int_{0}^{t} d \tau S_{t, \tau}\left(G_{\tau}\left(v_{\tau}-v_{\tau}^{\prime}\right)+\mathscr{N}_{\tau}\left(v_{\tau}\right)-\mathcal{N}_{\tau}\left(v_{\tau}^{\prime}\right)\right)
$$

Using the assumption $A_{N}^{\prime}$, this leads to a bound

$$
\begin{aligned}
b(t)\left\|(\mathscr{M} v)_{t}-\left(\mathscr{M} v^{\prime}\right)_{t}\right\| \leqq & b(t) \int_{0}^{t} d \tau s(t, \tau) g(\tau) b(\tau)^{-1}\left\|v_{\tau}-v_{\tau}^{\prime}\right\| \\
& +b(t) \int_{0}^{t} d \tau s(t, \tau) m(\tau) b(\tau)^{-v^{\prime}} A^{v^{\prime}} b(\tau)^{-1}\left\|v_{\tau}-v_{\tau}^{\prime}\right\|
\end{aligned}
$$

If we define the constant $M$ by

$$
M=\sup _{T \geqq t>0} b(t) \int_{0}^{t} d \tau s(t, \tau) m(\tau) b(\tau)^{-v^{\prime}-1}
$$

then we see that $\mathscr{M}$ will be a contraction of the ball of radius $A$ into itself provided

$$
\begin{gathered}
G+M A^{v^{\prime}}<1, \\
S\left\|v_{0}\right\|+F<1-G-M A^{v^{\prime}} .
\end{gathered}
$$

This is always possible if $(2.5)$ holds and $M,\left\|v_{0}\right\|, F$ are sufficiently small. Hence we have shown the

Theorem 2.1. If the inequalities (2.5), (2.6), (2.8) hold, then Eq. (2.1) has, for all sufficiently small initial data $v_{0} \in \mathscr{B}$ a unique solution for $t<T$. This solution will be bounded by

$$
\|v\| \| A .
$$

Remark 2.2. If $v_{0}=0$, then the condition (2.4) shows that if $G<1$, the constant $A$ can be chosen of order $\mathcal{O}(F)$. Note that if $F$, which is an integral $\int_{0}^{t}$, goes to zero like $t^{\gamma}$,
$\gamma>0$, as $t \rightarrow 0$, then this implies

$$
\left\|v_{t}\right\|=\mathcal{O}\left(t^{\gamma}\right)
$$

\section{Proof of Theorem 1.1}

We write the equation for $\delta_{t}$, which is the difference

$$
\delta_{t}=T_{t} v_{0}-S_{t} v_{0}
$$

We denote $w_{t}=u_{t}^{(1)}=S_{t} v_{0}$. Straightforward expansion leads to the equation

$$
\begin{gathered}
\partial_{t} \delta_{t}=\mathrm{L}_{t} \delta_{t}+\mathrm{F}_{t}+\mathscr{N}_{t}\left(\delta_{t}\right), \\
L_{t}=4 \partial_{x}^{2}+3-4 i \varepsilon \partial_{x}^{3}-\varepsilon^{2} \partial_{x}^{4}-3\left(2\left|w_{t}\right|^{2}+w_{t}^{2} J\right)-3 e^{2 i x / \varepsilon} w_{t}^{2},
\end{gathered}
$$

with

$$
\begin{gathered}
J f(x)=\bar{f}(x), \\
F_{t}=-w_{t}^{3} e^{2 i x / \varepsilon}-\left(4 i \varepsilon \partial_{x}^{3}+\varepsilon^{2} \partial_{x}^{4}\right) w_{t},
\end{gathered}
$$


and

$$
\mathscr{N}_{t}(\delta)=-3\left(2 w_{t}|\delta|^{2}+\bar{w}_{t} \delta^{2}+\delta|\delta|^{2}\right)-e^{2 i x / \varepsilon}\left(3 w_{t} \delta^{2}+\delta^{3}\right) .
$$

It is our aim to show that $\delta_{t}$, which starts out being 0 at time $t=0$, will grow relatively slowly. Note, however, that the terms

$$
4 \partial_{x}^{2}+3-6\left|w_{t}\right|^{2}-3 w_{t}^{2} J
$$

give rise to an operator which is independent of $\varepsilon$. This operator is just the tangent map to the flow defined by the semigroup $S_{t}$, and unless there is some cancellation, or a suitable renormalization of the "free" evolution $w_{t}$, we expect this term to give rise to an exponentially growing error, (starting from zero). All other terms in (3.1) will be seen to produce error-increasing "forces" which are of order $\varepsilon$. For those having an explicit factor of $\varepsilon$, this seems fairly obvious, and for the rapidly oscillating terms, this will follow by integration by parts. The difficulty in proving these intuitively obvious statements comes from the necessity to provide the right kind of regularity at the right point in the proof.

We next describe the steps through which the function $\delta_{t}$ will be estimated.

Step 1. We consider the "free" equation

$$
\partial_{t} u^{(1)}=4 \partial_{x}^{2} u^{(1)}+3 u^{(1)}-3 u^{(1)}\left|u^{(1)}\right|^{2} .
$$

One has the bound

Lemma 3.1. For every initial condition $u_{0}^{(1)}$ satisfying $\left\|\partial_{x}^{k} u_{0}^{(1)}\right\|_{\infty}<\infty$ for $k=0, \ldots, 4$, the norms $\left\|\partial_{x}^{k} u_{t}^{(1)}\right\|_{\infty}$ are uniformly bounded for all $t>0$ and $k=0, \ldots, 4$.

Remark. If we require only $\left\|u_{0}^{(1)}\right\|_{\infty}<\infty$, then for any $t>0$ all derivatives are bounded, but not uniformly as $t \rightarrow 0$.

This lemma will be proved in Appendix A.

Step 2. We next study the operator

$$
L^{(2)}=4 \partial_{x}^{2}-4 i \varepsilon \partial_{x}^{3}-\varepsilon^{2} \partial_{x}^{4}+3
$$

This is a part of the operator $L_{t}$ in (3.2). We have the following bound

Lemma 3.2. The operator $L^{(2)}$ is the generator of a semigroup $S_{t}^{(2)}=\exp \left(t L^{(2)}\right)$ which satisfies, for $r=0,1$,

$$
\left\|\partial_{x}^{r} S_{t}^{(2)} v\right\|_{\infty} \leqq a_{r}(t)\|v\|_{\infty}
$$

where

$$
a_{r}(t)=\text { const } \min \left(t^{-r / 2},\left(\varepsilon^{2} t\right)^{-r / 4}\right),
$$

uniformly in $t>0$.

Remark. If $v$ is differentiable, then we can write this in the more useful way

$$
\left\|S_{t}^{(2)} \partial_{x}^{r} v\right\|_{\infty} \leqq a_{r}(t)\|v\|_{\infty} .
$$

This lemma will be proved in Appendix B. 
Step 3. We next add the potential terms of (3.2):

$$
G_{t}^{(2)}=-3\left(2\left|u_{t}^{(1)}\right|^{2}+\left(u_{t}^{(1)}\right)^{2} J\right)-3 e^{2 i x / \varepsilon}\left(u_{t}^{(1)}\right)^{3} .
$$

We form a new semigroup $S_{t, t^{\prime}}^{(3)}$ which is the solution of

$$
S_{t, t^{\prime}}^{(3)}=S_{t-t^{\prime}}^{(2)}+\int_{t^{\prime}}^{t} d s S_{t-s}^{(2)} G_{s}^{(2)} S_{s, t^{\prime}}^{(3)}
$$

with the initial condition $S_{t^{\prime}, t^{\prime}}^{(3)}=1$. (For the semigroups defined below, we shall not repeat this condition.)

Lemma 3.3. There is $a T>0$ and a constant $C$ such that for $r=0,1$ one has the bound

$$
\left\|S_{t, t^{\prime}}^{(3)} \partial_{x}^{r} v\right\|_{\infty} \leqq C a_{r}\left(t-t^{\prime}\right)\|v\|_{\infty},
$$

when $0 \leqq t-t^{\prime}<T$.

Proof. This is the first application of Theorem 2.1. We begin with the case $r=0$. The Banach space will be $L^{\infty}$, and the weight function $b$ will be constant. There is only the original semigroup $S^{(2)}$ and a linear perturbation $G^{(2)}$. By Lemma 3.1, we see that for $0<t<T$, one has $\left\|G_{t}^{(2)}\right\|_{\infty}<G_{1}$. By Lemma 3.2, we see that $\left\|S_{t}^{(2)}\right\|_{\infty}<S_{1}$, for all $t>0$. Therefore the condition (2.4) reads in this case:

$$
T S_{1}\left\|v_{0}\right\|_{\infty}+T G_{1} A \leqq A .
$$

The condition on contractivity reads $T G_{1}<1$. Therefore, for sufficiently small $T>0$, the Theorem 2.1 applies and this yields the Lemma 3.3 for $r=0$.

In order to study the case $r=1$ we use the equation

$$
S_{t, t^{\prime}}^{(3)} \partial_{x}=S_{t-t^{\prime}}^{(2)} \partial_{x}-\int_{t^{\prime}}^{t} d s S_{t, s}^{(3)} G_{s}^{(2)} S_{s-t^{\prime}}^{(2)} \partial_{x}
$$

By the estimates of Lemma 3.1 and Lemma 3.2, we see that

$$
\left\|G_{s}^{(2)} S_{s-t^{\prime}}^{(2)} \partial_{x} w\right\|_{\infty} \leqq \text { const } a_{1}\left(s-t^{\prime}\right)\|w\|_{\infty} .
$$

Combining this with (3.10) for $r=0$, we conclude that

$$
\left\|\int_{t^{\prime}}^{t} d s S_{t, s}^{(3)} G_{s}^{(2)} S_{s-t^{\prime}}^{(2)} \partial_{x} w\right\|_{\infty} \leqq \operatorname{const}\left(t-t^{\prime}\right) \cdot a_{1}\left(t-t^{\prime}\right)\|w\|_{\infty} .
$$

The last expression is a bound on

$$
\text { const } \int_{t^{\prime}}^{t} d s a_{0}(t-s) a_{1}\left(s-t^{\prime}\right)
$$

which uses the explicit form of $a_{0}$ and $a_{1}$. Since the bound is dominated by that on $S_{t-t^{\prime}}^{(2)} \partial_{x}$, the proof of Lemma 3.3 is complete.

Step 4. We now add the other terms. We start with the inhomogeneous term

$$
F_{t}^{(3)}(x)=-e^{2 i x / \varepsilon}\left(u_{t}^{(1)}(x)\right)^{3}
$$


It can be rewritten as

$$
\begin{aligned}
F_{t}^{(3)}(x) & =-\frac{\varepsilon}{2 i}\left(u_{t}^{(1)}(x)\right)^{3} \partial_{x} e^{2 i x / \varepsilon} \\
& =-\frac{\varepsilon}{2 i} \partial_{x}\left(F_{t}^{(3)}(x)\right)+\frac{\varepsilon}{2 i} e^{2 i x / \varepsilon} \partial_{x} F_{t}^{(3,2)}(x)
\end{aligned}
$$

where $F_{t}^{(3,2)}=\left(u_{t}^{(1)}\right)^{3}$. We define further

$$
\varepsilon F_{t}^{(3,3)}(x)=-\left(4 i \varepsilon \partial_{x}^{3}+\varepsilon^{2} \partial_{x}^{4}\right) u_{t}^{(1)}(x),
$$

which is the second term in (3.3). We have the following bounds, using Lemma 3.1:

$$
\begin{gathered}
\left\|\partial_{x}^{r} F_{t}^{(3,2)}\right\|_{\infty}<F_{1}, \\
\left\|F_{t}^{(3,3)}\right\|_{\infty}<F_{2}, \\
\left\|F_{t}^{(3)}\right\|_{\infty}<F_{3},
\end{gathered}
$$

uniformly in $\varepsilon$, for $0 \leqq t<T$. Note that $\left\|\partial_{x} F_{t}^{(3)}\right\|_{\infty}$ diverges like $\mathcal{O}(1 / \varepsilon)$ as $\varepsilon \rightarrow 0$.

Finally, we define the nonlinearity $\mathscr{N}^{(3)}$ as in (3.4) by

$$
\begin{aligned}
\mathscr{N}_{t}^{(3)}(\delta)(x)= & -3\left(2 u_{t}^{(1)}(x)|\delta(x)|^{2}+\bar{u}_{t}^{(1)}(x) \delta^{2}(x)+\delta(x)|\delta(x)|^{2}\right) \\
& -e^{2 i x / \varepsilon}\left(3 u_{t}^{(1)}(x) \delta^{2}(x)+\delta^{3}(x)\right) .
\end{aligned}
$$

With this notation, $\delta$ is given by the expression

$$
\delta_{t}=\int_{0}^{t} d s S_{t, s}^{(3)}\left(F_{s}^{(3)}+\varepsilon F_{s}^{(3,3)}+\mathcal{N}_{s}^{(3)}\left(\delta_{t}\right)\right)
$$

which by (3.14) can be rewritten as

$$
\delta_{t}=\frac{\varepsilon}{2 i} \int_{0}^{t} d s\left(\left(S_{t, s}^{(3)} \partial_{x}\right) F_{s}^{(3)}+S_{t, s}^{(3)}\left(e^{2 i x / \varepsilon} \partial_{x} F_{s}^{(3,2)}+2 i F_{s}^{(3,3)}\right)\right)+\int_{0}^{t} d s S_{t, s}^{(3)} \mathcal{N}_{s}^{(3)}\left(\delta_{s}\right)
$$

By the bounds we have established on $S^{(3)}$, and on $S^{(3)} \partial_{x}$, we see that the main lemma applies for sufficiently small $T>0$. The corresponding bounds for (2.3) are:

$$
\begin{gathered}
F \leqq \text { const } \min \left(\varepsilon t^{1 / 2}, \varepsilon^{1 / 2} t^{3 / 4}\right), \\
G=0, \\
\left\|v_{0}\right\|=0, \\
M, N \leqq \text { const } t .
\end{gathered}
$$

These constants depend only on the first four derivatives of $u_{0}^{(1)}$. Hence $\delta_{t}$ exists for $0 \leqq t<T$. Applying the Remark 2.2, the assertion of Theorem 1.1 follows.

\section{Appendix A}

In this section, we prove Lemma 3.1. However, since our method is more general, we present a general bound on solutions of semilinear differential equations, which need not be parabolic. The idea is that the nonlinearity bounds those terms which 
are not damped by the positivity of the "kinetic" energy. We consider equations of the form

$$
\partial_{t} u_{t}=-P u_{t}-u_{t} f\left(\left|u_{t}\right|^{2}\right),
$$

where $P$ is an elliptic differential operator, or, more generally associated to a symbol

$$
P(x, \xi)=a \xi^{2 k}+R(x, \xi),
$$

where $a>0$ and where $R$ is a polynomial of degree $<2 k$ in $\xi$ with sufficiently smooth coefficients which are bounded together with their derivatives.

We also assume that $f(\varrho) \rightarrow \infty$ as $\varrho \rightarrow \infty$ and $f^{\prime}(\varrho) \geqq 0$. This last condition is somewhat too restrictive but makes the estimates easier. We denote by $P$ the operator $P=P\left(x,-i \partial_{x}\right)$. The method we use generalizes immediately to operators in several variables, when $a$ is a definite form.

To make the notation less awkward, we pretend in all estimates that the quantities are real, thus we write $u^{2}$ instead of $|u|^{2}$. The necessary adaptations to the complex, or general vector-valued case are obvious.

Theorem 4.1. Assume $\left\|u_{0}\right\|_{\infty}<\infty$ and $\left\|\partial_{x} u_{0}\right\|_{\infty}<\infty$. Under the above assumptions on $P$ and $f$, the solutions of (4.1) are bounded in $L^{\infty}$. There is a $C=C\left(\left\|u_{0}\right\|_{\infty},\left\|\partial_{x} u_{0}\right\|_{\infty}\right)<\infty$ such that

$$
\left\|u_{t}\right\|_{\infty} \leqq C\left(\left\|u_{0}\right\|_{\infty},\left\|\partial_{x} u_{0}\right\|_{\infty}\right) .
$$

Remark. By the standard regularity theorems, there is, for every $p$, a $T>0$, and a $C>0$, such that $\left\|\partial_{x}^{p} u_{t}\right\|_{\infty}<C$ for all $t \in(0, T)$, and for $p \leqq p_{0}$. We may thus assume that the initial data are in $\mathscr{C}^{p_{0}}$, for any $p_{0}<\infty$ we wish.

Proof. Our strategy will be to prove the result first in Sobolev spaces and then derive the theorem from the Sobolev estimate. We give ourselves a weight function $h \in \mathscr{C}^{2 k}, h^{2} \in L^{2}, h>0$ which satisfies

$$
\left\|\partial_{x}^{p} h / h\right\|_{\infty} \leqq H, \text { for } p=1, \ldots, 2 k .
$$

We shall do bounds in $L^{2}\left(h^{2} d x\right)$ and denote

$$
(u, v)_{h}=\int d x h^{2}(x) \bar{u}(x) v(x)
$$

and

$$
(u, v)=\int d x \bar{u}(x) v(x) .
$$

Remark. The class of functions $h$ which we really use is

$$
h(x)=\left(1+a_{0}\left(x-a_{1}\right)^{2}\right)^{-1},
$$

with $a_{0}>0$ fixed, and $a_{1}$ arbitrary. Then the bounds (4.3) hold with $H$ independent of $a_{1}$.

Let $Q(x, \xi)$ be of the form

$$
Q(x, \xi)=b \xi^{2 n}+S(x, \xi),
$$

where $b>0$ and where $S$ is a polynomial of degree $<2 n$ in $\xi$ with sufficiently smooth coefficients which are bounded together with their derivatives. We denote by $Q$ the operator $Q\left(x,-i \partial_{x}\right)$. 
Lemma 4.2. There is a constant $G=G(b, S, H)$ such that

$$
(u, Q u)_{h} \geqq-G(u, u)_{h} .
$$

Proof. We have

$$
(u, Q u)_{h}=\left(h^{2} u, Q u\right)=(u h, Q u h)+\left(u h,[h, Q] h^{-1} \cdot u h\right),
$$

where $[\cdot, \cdot]$ denotes the commutator. Note now that $\left[h, \partial_{x}^{p}\right] h^{-1}$ is a sum of differential operators of degree less than $p$,

$$
\left[h, \partial_{x}^{p}\right] h^{-1}=\sum_{\substack{j_{1}, \ldots, j_{s} \\ 1 \leqq \Sigma j_{k} \leqq p}} c_{j_{1}, \ldots, j_{s}} \prod_{k=1}^{s}\left(\frac{\partial_{x}^{j_{k}} h}{h}\right) \partial_{x}^{p-\Sigma j_{k}}
$$

Therefore, we find

$$
[h, Q] h^{-1}=\sum_{j=1}^{2 n} S_{j}\left(x,-i \partial_{x}\right),
$$

where the $S_{j}$ are polynomials of degree at most $2 n-j$ in the second argument, whose coefficients are bounded when $h$ is of the form of (4.4). By our assumptions, we find that

$$
Q_{0}(x, \xi)=Q(x, \xi)+\sum_{j=1}^{2 n} S_{j}(x, \xi)
$$

is a symbol of order $2 n$ and it satisfies

$$
Q_{0}(x, \xi) \geqq-K
$$

for some $K=K(b, S, H)$. Therefore, we have

$$
\left(u h^{2}, Q u\right)=\left(u h, Q_{0}\left(x,-i \partial_{x}\right) u h\right) \geqq-G(u h, u h)
$$

for some $G=G(b, S, H)$, by [3, Theorem 18.1.14-15]. This completes the proof of Lemma 4.2.

We consider next an initial condition $u_{0} \in L^{\infty}$ and establish a bound on $\left(u_{t}, u_{t}\right)_{h}$.

Proposition 4.3. There is a constant $C_{1}=C_{1}\left(a, R, H,\left\|u_{0}\right\|_{\infty}\right)$ such that

$$
\int d x h^{2}(x) u_{t}^{2}(x) \leqq C_{1} \text {. }
$$

Proof. Consider

$$
\begin{aligned}
\frac{1}{2} \partial_{t}\left(u_{t}, u_{t}\right)_{h} & =\left(u_{t}, \partial_{t} u_{t}\right)_{h} \\
& =-\left(u_{t}, P u_{t}\right)_{h}-\left(u_{t}, u_{t} f\left(u_{t}^{2}\right)\right)_{h} .
\end{aligned}
$$

By the lemma, this quantity is bounded by

$$
\frac{1}{2} \partial_{t}\left(u_{t}, u_{t}\right)_{h} \leqq G\left(u_{t}, u_{t}\right)_{h}-\left(u_{t}, u_{t} f\left(u_{t}^{2}\right)\right)_{h} .
$$

Define now $U$ such that $f(U) \geqq G+1$. Then

$$
\begin{aligned}
\frac{1}{2} \partial_{t}(u, u)_{h} & \leqq G \int h^{2} u^{2}-\int_{u^{2} \geqq U} h^{2} u^{2}(G+1) \\
& \leqq G \int_{u^{2} \leqq U} h^{2} u^{2}-\int_{u^{2} \geqq U} h^{2} u^{2} \\
& \leqq(G+1) \int_{u^{2} \leqq U} h^{2} u^{2}-\int h^{2} u^{2} .
\end{aligned}
$$


Thus, $\frac{1}{2} \partial_{t}(u, u)_{h} \leqq(G+1) U-(u, u)_{h}$. From this differential inequality it follows at once that

$$
\left(u_{t}, u_{t}\right)_{h} \leqq \max \left(2(G+1) U,\left(u_{0}, u_{0}\right)_{h}\right)
$$

We next bound

$$
\begin{aligned}
\frac{1}{2} \partial_{t}\left(u^{\prime}, u^{\prime}\right)_{h}= & -\left(u^{\prime}, P u^{\prime}\right)_{h}-\left(u^{\prime}, f\left(u^{2}\right) u^{\prime}\right)_{h} \\
& -2\left(u u^{\prime}, f^{\prime}\left(u^{2}\right) u u^{\prime}\right)_{h} .
\end{aligned}
$$

Note that the terms containing $f$ are non-positive. Assume for simplicity that $P(x, \xi)$ in (4.2) only depends on $\xi$. Fix any $A \in \mathbf{R}$. Then the identity

$$
\left(u^{\prime},(P-A) u^{\prime}\right)_{h}=(u, Q u)_{h},
$$

holds when

$$
Q(x, \xi)=\left(\xi^{2}-2 i \frac{h^{\prime}}{h}(x) \xi\right)(P(\xi)-A) .
$$

Remark. When $P$ is of the general form of (4.2), one can still find a $Q$ such that (4.6) holds. The definition of $Q$ is more complicated, but the terms of highest order remain the same in all cases.

We now apply Lemma 4.2 with $Q$ as above and get

$$
(u, Q u)_{h} \geqq-C(u, u)_{h} .
$$

Therefore the identity (4.6) implies

$$
\left(u^{\prime}, P u^{\prime}\right)_{h}-A\left(u^{\prime}, u^{\prime}\right)_{h}=(u, Q u) \geqq-C(u, u)_{h} .
$$

It follows that

$$
\frac{1}{2} \partial_{t}\left(u^{\prime}, u^{\prime}\right)_{h} \leqq-\left(u^{\prime}, P u^{\prime}\right)_{h} \leqq C(u, u)_{h}-A\left(u^{\prime}, u^{\prime}\right)_{h} .
$$

We have already seen that $\left(u_{t}, u_{t}\right)_{h}$ is bounded, and hence it follows that $\left(u_{t}^{\prime}, u_{t}^{\prime}\right)$ is bounded as well.

We now have shown that $\left(u_{t}, u_{t}\right)_{h}$ and $\left(u_{t}^{\prime}, u_{t}^{\prime}\right)_{h}$ are bounded in $t$. From this the proof of the theorem is completed as follows. We have

$$
\begin{aligned}
h^{2}(x) u^{2}(x) & =\int_{-\infty}^{x} d y\left(u^{2} h^{2}\right)^{\prime}(y) \\
& =2 \int_{-\infty}^{x} d y u u^{\prime} h^{2}+2 \int_{-\infty}^{x} d y u^{2} \frac{h^{\prime}}{h} h^{2} \\
& \leqq 2(u, u)_{h}^{1 / 2}\left(u^{\prime}, u^{\prime}\right)_{h}^{-1 / 2}+2(u, u)_{h}\left\|\frac{h^{\prime}}{h}\right\|_{\infty}<\infty .
\end{aligned}
$$

Thus, we find

$$
|u(x)| \leqq K h(x)^{-1},
$$

but since $K$ is independent of $h$ among the $h$ of (4.4), we find, using $a_{1}=x$,

$$
|u(x)| \leqq K \text {. }
$$


Proof of Lemma 3.1. To simplify the notation, consider

$$
\partial_{t} u=4 \partial_{x}^{2} u+3 u-3 u|u|^{2}=4 \partial_{x}^{2} u+f(u)
$$

and denote by $u^{(k)}$ the $k^{\text {th }}$ derivative of $u$. Let

$$
\phi_{k}(t)=\sup _{0 \leqq t \leqq T} \sup _{0 \leqq p \leqq k}\left\|\partial_{x}^{p} u_{t}\right\|_{\infty} .
$$

We are going to show that if $\phi_{k}(0)<\infty$, then $\phi_{k}(t)<\infty$ for all $t>0$. In fact, one can even gain one derivative with a slight divergence near $t=0$, but we do not need these better bounds in this paper.

It is well known from local existence and regularity theorems that there is a $T=T\left(\phi_{k}(0)\right)>0$ such that $\phi_{k}(T)<\infty$. So all we have to show is $\phi_{k}(t)<\infty$ for $t>T$. This will follow from the regularizing properties of the heat kernel. Indeed, if we denote by $G_{t}$ the heat kernel corresponding to the operator $\partial_{t} u=4 \partial_{x}^{2} u$, then we have

$$
\left\|\partial_{x} G_{t}\right\|_{1} \leqq \mathcal{O}\left(t^{-1 / 2}\right),
$$

where the $L^{1}$ norm is in the space variable. For $t>T$, the evolution equation leads to

$$
u_{t}^{(k)}=\left(\partial_{x} G_{T}\right) * u_{t-T}^{(k-1)}+\int_{0}^{T} d s\left(\partial_{x} G_{T-s}\right) * f\left(u_{t-T+s}^{(k-1)}\right) .
$$

Here, $*$ denotes convolution. In terms of the bounds, we get

$$
\phi_{k}(t) \leqq\left\|\partial_{x} G_{T}\right\|_{1} \phi_{k-1}(t-T)+\int_{0}^{T} d s\left\|\partial_{x} G_{s}\right\|_{1} R_{k-1}(s),
$$

where $R_{k-1}$ is a polynomial in $\phi_{l}$, with $l<k$. From the bound on $\partial_{x} G$, we obtain a recursion relation of the form

$$
\phi_{k}(t) \leqq \operatorname{const}\left(T^{-1 / 2}+\int_{0}^{T} d s s^{-1 / 2}\right) F_{k-1}\left(\phi_{k-1}(t)\right),
$$

for some polynomial $F_{k-1}$. Since we have already shown $\phi_{0}(t)<\infty$ for all $t>0$, the result follows for all $k$ by induction.

\section{Appendix B, Proof of Lemma 3.2}

To simplify the notation, we consider instead of $L^{(2)}$ the operator

$$
\partial_{x}^{2}-i \varepsilon \partial_{x}^{3}-\varepsilon^{2} \partial_{x}^{4} .
$$

We consider the kernel $G$ of the corresponding semigroup. It is defined by

$$
G_{t}(x)=\int_{-\infty}^{\infty} d k e^{i k x} e^{-t\left(k^{2}+i \varepsilon k^{3}+\varepsilon^{2} k^{4}\right)} .
$$

One has the following bound.

Proposition 5.1. For all $t>0$ and all $r \geqq 0$ one has

$$
\left\|\partial_{x}^{r} G_{t}\right\|_{1} \leqq \text { const } \min \left(t^{-r / 2},\left(\varepsilon^{2} t\right)^{-r / 4}\right) .
$$

Remark. Clearly, this implies Lemma 3.2. 
Proof. We shall call (a) and (b) the two alternatives in (5.1). It is useful to consider the rescaled function

$$
g_{\tau}(y)=\int_{-\infty}^{\infty} d k e^{i k y} e^{-k^{2}-i \tau k^{3}-\tau^{2} k^{4}}
$$

so that $G_{t}(x)=t^{-1 / 2} g_{\tau}\left(x / t^{1 / 2}\right)$ with $\tau=\varepsilon t^{-1 / 2}$. This will allow us to treat the case $\tau \rightarrow 0$.

Lemma 5.2. For all $\alpha, \beta \geqq 0$ there is a constant $C_{\alpha, \beta}$ such that for all $\tau, 0 \leqq \tau \leqq 1$, one has

$$
\left\|\left(1+y^{2 \alpha}\right) \partial_{y}^{\beta} g_{\tau}(y)\right\|_{\infty}<C_{\alpha, \beta} .
$$

Proof. This follows by direct calculation. We have

$$
\begin{aligned}
\left(1+y^{2 \alpha}\right) \partial_{y}^{\beta} g_{\tau}(y) & =\int_{-\infty}^{\infty} d k e^{i k y}\left(1+\left(-i \partial_{k}\right)^{2 \alpha}\right)(i k)^{\beta} e^{-k^{2}-i \tau k^{3}-\tau^{2} k^{4}} \\
& =\int_{-\infty}^{\infty} d k e^{i k y} P_{\alpha, \beta}(k) e^{-k^{2}-i \tau k^{3}-\tau^{2} k^{4}}
\end{aligned}
$$

Here, $P_{\alpha, \beta}$ is a polynomial whose coefficients are uniformly bounded for $\tau \in[0,1]$. The assertion follows.

Taking $\alpha=1$ in Lemma 5.2 and integrating over $y$, the bound (a) of Proposition 5.1 follows for $\tau=\varepsilon t^{-1 / 2}<1$.

We introduce a second rescaling: $g_{\tau}(y)=\tau^{-1 / 2} h\left(\tau^{-1 / 2} y\right)$.

Lemma 5.3. For all $\alpha, \beta \geqq 0$ there is a constant $C_{\alpha, \beta}$ such that for all $\tau, \tau \geqq 1$, one has

$$
\left\|\left(1+z^{2 \alpha}\right) \partial_{z}^{\beta} h_{\tau}(z)\right\|_{\infty}<C_{\alpha, \beta} .
$$

Proof. We can rewrite $h_{\tau}$ as

$$
h_{\tau}(z)=\int_{-\infty}^{\infty} d q e^{i q z} e^{-\eta^{2} q^{2}-i \eta q^{3}-q^{4}}
$$

where now $\eta=\tau^{-1 / 2}=\varepsilon^{-1 / 2} t^{1 / 4}$. Since $\tau \geqq 1$ implies $\eta \leqq 1$, the proof now follows the same steps as in the case of Lemma 5.2.

If we re-express the results obtained so far in terms of $G$, we see that

$$
G_{t}(y)=\theta^{-1} h_{\tau}\left(\theta^{-1} y\right),
$$

with $\theta=\varepsilon^{1 / 2} t^{1 / 4}$, so that Lemma 5.3 implies the bound (b) for $\varepsilon t^{-1 / 2}>1$. In particular, when $r=0$ then (a) and (b) together imply

$$
\left\|G_{t}\right\|_{1} \leqq \text { const, uniformly in } t>0 \text {. }
$$

We now prove the bounds of Proposition 5.1 for $r \geqq 1$. The result will follow essentially from scaling. We consider the case $\alpha=1$, only. Then the polynomial $P_{1, \beta}$ is of the form

$$
\begin{gathered}
(i k)^{\beta}+\beta(\beta-1)(i k)^{\beta-2}+2 i \beta(i k)^{\beta-1}\left(2 k+3 i \tau k^{2}+4 \tau^{2} k^{3}\right) \\
-(i k)^{\beta}\left(2+6 i \tau k+12 \tau^{2} k^{2}\right)-(i k)^{\beta}\left(2 k+3 i \tau k^{2}+4 \tau^{2} k^{3}\right)^{2}
\end{gathered}
$$


Note now that, for all $n, m \geqq 0$,

$$
\begin{aligned}
& \left|k^{2}\right|^{m} e^{-k^{2} / 2}<C_{m}, \\
& \left|\tau^{2} k^{4}\right|^{n} e^{-\tau^{2} k^{4}}<C_{n} .
\end{aligned}
$$

We see that for $\beta \geqq 1$ all the terms in (5.3) can be bounded by expressions of the form const $\left|k^{2}\right|^{m}\left|\tau^{2} k^{4}\right|^{n}$ with non-negative $m, n$. Therefore

$$
\left|P_{1, \beta}(k) e^{-\left(k^{2}+i \tau k^{3}+\tau^{2} k^{4}\right) / 2}\right|<C(\beta),
$$

uniformly in $k$ and $\tau$. Using the remaining part of the exponential to bound the integration, we see that

$$
\left\|\left(1+y^{2}\right) \partial_{y}^{\beta} g_{\tau}(y)\right\|_{\infty}<\text { const } C_{\beta},
$$

for all $\beta \geqq 1$. This proves part (a) of Proposition 5.1. A similar calculation for $h$ leads to the polynomial

$$
\begin{gathered}
(i q)^{\beta}+\beta(\beta-1)(i q)^{\beta-2}+2 i \beta(i q)^{\beta-1}\left(2 \eta^{2} q+3 i \eta q^{2}+4 q^{3}\right) \\
-(i q)^{\beta}\left(2 \eta^{2}+6 i \eta q+12 q^{2}\right)-(i q)^{\beta}\left(2 \eta^{2} q+3 i \eta q^{2}+4 q^{3}\right)^{2} .
\end{gathered}
$$

The desired bound follows now as before by observing that

$$
\begin{gathered}
\left|\eta^{2} q^{2}\right|^{m} e^{-\eta^{2} q^{2} / 2}<C_{m}, \\
\left|q^{4}\right|^{n} e^{-q^{4} / 2}<C_{n},
\end{gathered}
$$

and checking that all terms in (5.4) can be bounded by terms with non-negative $m$ and $n$. The proof of Proposition 5.1 can now be completed easily.

Acknowledgements. This work was supported by the Fonds National Suisse and IHES, Paris.

\section{References}

1a. Collet, P., Eckmann, J.-P.: Instabilities and fronts in extended systems. Princeton: Princeton University Press 1990

1b. Collet, P., Eckmann, J.-P.: The existence of dendritic fronts. Commun. Math. Phys. 107, 39-92 (1986)

1c. Collet, P., Eckmann, J.-P.: The stability of modulated fronts. Helv. Phys. Acta 60, 969-991 (1987)

2. Eckmann, J.-P., Wayne, C.E.: Propagating fronts and the center manifold theorem. Preprint

3. Lang, S.: Introduction to differentiable manifolds. New York: Wiley 1962

4. Hörmander, L.: The analysis of linear partial differential operators. Berlin, Heidelberg, New York: Springer 1983-1985

Communicated by A. Jaffe

Received January 2, 1990; in revised form March 16, 1990 
